\title{
ДИАГНОСТИКА И ЛЕЧЕНИЕ ОСТРЫХ ХИРУРГИЧЕСКИХ ЗАБОЛЕВАНИЙ У ПАЦИЕНТОВ С COVID-19
}

\author{
В. И. Вечорко, В. Д. Аносов, Б. В. Силаев
}

Городская клиническая больница № 15 имени О. М. Филатова, Москва, Россия

\begin{abstract}
Экстренная хирургическая помощь в условиях инфекционного стационара - актуальная проблема во время пандемии новой коронавирусной инфекции COVID-19. Городская клиническая больница № 15 имени О. М. Филатова оказывает экстренную хирургическую помощь в условиях перепрофилирования с 27 марта 2020 г. по настоящее время. Специалистами больницы накоплен большой практический опыт: в апреле 2020 г. выполнено 194 , а в мае 289 оперативных пособий. В статье представлен опыт работы экстренной хирургической службы на этапе перепрофилирования в инфекционный стационар. Среди всех госпитализированных пациентов 482 (5,29\%) имели острую хирургическую патологию, потребовавшую экстренного оперативного вмешательства. У 472 (98\%) экстренно прооперированных пациентов присутствовала вызванная COVID-19 внебольничная пневмония разной степени тяжести. В статье рассмотрены некоторые особенности острой хирургической патологии и осложнения, встречающиеся у пациентов с COVID-19. Особенности хирургической помощи в перепрофилированном стационаре - это строгое соблюдение эпидемиологического режима, минимизация численности персонала в операционной, возможная минимизация числа оперативных пособий и сокращение их длительности. Наиболее важной задачей в условиях пандемии COVID-19 является безопасность персонала.
\end{abstract}

Ключевые слова: острая хирургическая патология, перитонит, острая кишечная непроходимость, кровотечение, острый аппендицит, острый холецистит, грыжи, острый панкреатит, COVID-19, мезентериальный тромбоз

Вклад авторов: все авторы внесли равный вклад в планирование исследования, организацию работы стационара, сбор и обобщение материала, а также написание статьи.

Соблюдение этических стандартов: все пациенты подписали согласие на обработку персональных данных, а также согласие на оперативное лечение. В случае невозможности получения инсормированного согласия пациента ввиду тяжести его состояния, В соответствии с требованиями этического комитета и действующих нормативных актов был оформлен консилиум.

$\bowtie$ Для корреспонденции: Борислав Владимирович Силаев ул. Вешняковская, д. 23, г. Москва, 111539; drsilaev@yandex.ru

Статья получена: 15.06.2020 Статья принята к печати: 22.06.2020 Опубликована онлайн: 29.06.2020

DOI: $10.24075 /$ vrgmu.2020.038

\section{DIAGNOSIS AND TREATMENT OF ACUTE SURGICAL DISEASES IN PATIENTS WITH COVID-19}

Vechyorko VI, Anosov VD, Silaev BV凶

Municipal Clinical Hospital № 15 named after O. M. Filatov, Moscow, Russia

Emergency surgery in the infectious diseases hospital is an urgent problem during the COVID-19 pandemic. Municipal Clinical Hospital No.15 named after O. M. Filatov has been providing emergency surgical care after conversion, from March 27, 2020 until now. The hospital's medical staff has built up extensive experience: 194 surgical procedures were carried out in April, and 289 surgical procedures were carried out in May 2020. The paper reports the experience of emergency surgery at the stage of conversion to an infectious diseases hospital. Among all hospitalized patients, 482 (5.29\%) people had acute surgical pathology requiring emergency surgery. Among patients who underwent urgent surgery, 472 (98\%) people had the caused by COVID-19 community-acquired pneumonia of various degrees of severity. The paper discusses some features of acute surgical pathology and complications identified in patients with COVID-19. The surgical care features in the hospital after conversion are proper epidemiological regime implementation, minimization of the number of staff in the operating room, possible minimization of the number and reduction of the duration of surgical procedures. The most important challenge during the COVID-19 pandemic is medical staff safety.

Keywords: acute surgical pathology, peritonitis, acute intestinal obstruction, bleeding, acute appendicitis, acute cholecystitis, hernias, acute pancreatitis, COVID-19, mesenteric thrombosis

Author contribution: all authors made an equal contribution to study planning, hospital management, data acquisition and summarizing, as well as to manuscript writing.

Compliance with ethical standards: all patients submitted the informed consent to personal data processing and surgical treatment. When it was not possible to obtain the patient's informed consent due to the severity of the disease, a consultation was issued in accordance with the Ethics Committee requirements and local regulations.

$\triangle$ Correspondence should be addressed: Borislav V. Silaev Veshnyakovskaya, 23, Moscow, 111539; drsilaev@yandex.ru

Received: 15.06.2020 Accepted: 22.06.2020 Published online: 29.06.2020

DOI: $10.24075 /$ brsmu.2020.038

Вспышка коронавирусной инфекции в 2019 г. (COVID-19) бросила серьезный вызов всему человечеству [1]. Повсеместное введение режима самоизоляции и социального дистанцирования привело к прекращению роста заболеваемости, однако говорить о полном контроле над ситуацией пока рано.

В условиях пандемии нагрузка на хирургические службы существенно возросла [2-5]. Мировые хирургические сообщества (Society of American Gastrointestinal and Endoscopic Surgeons (SAGES) [6, 7], American College of Surgeons [8], Royal College of Surgeons of England [9]), а также российские общества хирургов [10], урологов и акушеров-гинекологов опубликовали совместные рекомендации по работе хирургической службы в новых условиях $[10,11]$. Основные положения «Временных методических рекомендаций» направлены, во-первых, на предотвращение заражения медицинского персонала, и, во-вторых, на разработку принципов оказания инфицированным пациентам медицинской помощи, которую нельзя отложить до окончания пандемии. Согласно «Временным методическим рекомендациям»:

- все плановые оперативные вмешательства, манипуляции и исследования должны быть отложены;

- при проведении экстренных медицинских процедур 
следует учитывать факт возможного инфицирования медицинских работников, поэтому объем вмешательства необходимо по возможности минимизировать;

- в лечебном процессе должно быть задействовано минимально необходимое количество персонала; следует максимально использовать возможности дистанционного общения между лечащей стороной и пациентом, а также дистанционные консультации специалистов;

- вполне вероятен десицит кадров в хирургической службе, так как хирурги могут быть призваны оказывать медицинскую помощь пациентам в инфекционных стационарах.

Целью настоящей работы было представить результаты диагностики и лечения острых хирургических заболеваний в условиях пандемии новой коронавирусной инфекции COVID-19.

\section{ПАЦИЕНТЫ И МЕТОДЫ}

C 27 марта 2020 г. после перепрофилирования, занявшего около пяти суток, ГКБ № 15 имени О. М. Филатова приступила к приему пациентов с COVID-19.

В короткие сроки был построен шлюз с каналами для входа, переодевания персонала в защитную одежду и выхода из «красной зоны».

Хирургическая служба была сокращена до минимума, так как большинство врачей всех специальностей, в том числе врачи хирургического просиля, были призваны оказывать помощь пациентам с COVID-19. Прием плановых пациентов был прекращен. Наряду с пациентами инфекционного профиля, в госпиталь стали поступать пациенты с острой хирургической патологией, травмами, острой урологической и гинекологической патологией на фоне COVID-19.

Несмотря на проблемы, экстренная хирургическая помощь должна была быть оказана всем пациентам своевременно, качественно и в полном объеме. Для решения этой задачи основополагающим было соблюдение следующих условий:

а) правильная маршрутизация больных;

б) выбор оптимального (чаще минимального) объема операции;

в) создание безопасных условий для работы операционной бригады.

K настоящему моменту опыт, накопленный уже столкнувшимися с новой инфекцией странами, позволил выработать принципы проведения операций в условиях пандемии COVID-19. Эти принципы в полном объеме реализованы в ГКБ №15:

- режим работы вентиляции операционного блока изменен в сторону преобладания вытяжки над притоком и создания отрицательного давления в операционной;

- весь хирургический персонал заходит в операционный блок в средствах индивидуальной защиты (СИЗ): защитный комбинезон, шапочка, медицинские очки или пластиковый щиток, бахилы, латексные перчатки и фильтрующий респиратор;

- помимо СИЗ хирурги и операционные медсестры надевают одноразовую стерильную операционную одежду и стерильные перчатки;

- пациенту надевают одноразовую шапочку и одноразовую хирургическую маску;

- в операционной поддерживают оптимальную температуру и влажность (с учетом того, что члены операционной бригады одеты в двойной комплект одежды);

- максимально используется одноразовый хирургический инструментарий;

- в операционной находятся только члены операционной бригады, во время операции двери предоперационной и операционной плотно закрыты;

- ответственные за доставку инструментария дежурные медсестры находятся в предоперационной;

- переговоры между операционным залом и вспомогательными службами проходят только по внутренней связи.

Все операции были выполнены в условиях соблюдения эпидемиологического режима предварительно обученным и проинструктированным персоналом с соблюдением правил асептики и антисептики.

С 1 апреля по 23 мая 2020 г. в ГКБ № 15 было прооперировано 482 пациента, что составило 5,29\% от общего числа поступивших в стационар пациентов. Среди пациентов с хирургическими патологиями было 226 мужчин (46,8\%) и 256 женщин (53,2\%), средний возраст пациентов составил 57,2 \pm 6,9 лет (табл. 1).

У 436 (90,4\%) пациентов наличие вируса было подтверждено результатами лабораторных исследований (назо-фарингеальный мазок, ПЦР). У остальных 46 (9,6\%) пациентов отсутствовало лабораторное подтверждение наличия вируса, однако все они имели четкую картину пневмонии вирусной этиологии высокой степени вероятности.

Пневмония вирусной этиологии высокой степени вероятности (различной степени тяжести) была обнаружена у 472 пациентов (97,9\%). Распределение больных по степени тяжести вирусной пневмонии отражено в табл. 2.

Критерии включения в исследование: пациенты обоего пола, любого возраста, с доказанной посредством ПЦР новой коронавирусной инфекцией COVID-19, выявленной острой хирургической патологией, требующие неотложной хирургической помощи. Критерии исключения: недоказанная новая коронавирусная инфекция COVID-19 у пациентов обоих полов.

Статистическую обработку данных проводили с использованием стандартных методов и приложения Microsoft Excel (Microsoft; CШA).

Таблица 1. Распределение пациентов по возрасту

\begin{tabular}{|l|c|c|}
\hline \multicolumn{1}{|c|}{ Возраст } & Число пациентов & \% от общего числа пациентов \\
\hline $18-44$ лет & 107 & 22,3 \\
\hline $44-60$ лет & 192 & 21,5 \\
\hline $60-75$ лет & 104 & 15,1 \\
\hline $75-90$ лет & 73 & 1,2 \\
\hline$>90$ лет & 6 & 100 \\
\hline Всег: & 482 & \\
\hline
\end{tabular}




\section{РЕЗУЛЬТАТЫ ИССЛЕДОВАНИЯ}

Характер и количество операций, выполненных в клинике в период с 1 апреля по 23 мая 2020 г., представлены в табл. 3.

Из всех прооперированных пациентов только 32 (6,6\%) были помещены в реанимационные отделения. Остальных после пробуждения транспортировали в палату непосредственно из операционной.

\section{ОБСУЖДЕНИЕ РЕЗУЛЬТАТОВ}

C учетом полученных данных, необходимо подробнее остановиться на особенностях диагностики и лечения некоторых острых хирургических заболеваний в условиях перепрофилирования в инфекционный стационар для пациентов с COVID-19.

До перепрофилирования в инфекционный стационар диагностически «неясным» больным с подозрением на острый аппендицит назначали мультиспиральную компьютерную томографию (МСКТ) брюшной полости, но в условиях перегруженности кабинетов КТ стали чаще использовать диагностические возможности лапароскопии. Так, из 36 пациентов с подозрением на острый аппендицит во время диагностической лапароскопии диагноз был подтвержден у 28.

В некоторых публикациях рекомендуют выполнение открытых, а не лапароскопических операций, так как считается, что во время лапароскопической процедурь увеличивается риск инфицирования персонала [7, 8]. Другие специалисты выполняют аппендэктомию лапароскопическим доступом даже у пациентов с вирусной пневмонией [12]. Мы не стали менять привычную хирургическую тактику. В период перепрофилирования было выполнено 27 лапароскопических аппендэктомий. Длительность операции составила в среднем 44,7 мин (для сравнения, средняя длительность 25 лапароскопических аппендэктомий, выполненных в январе-феврале 2020 г., составляла 42,4 мин). В одном случае мы прибегли к лапаротомии и выполнили аппендэктомию «открытым» способом. Осложнений в послеоперационном периоде не было.

В литературе обсуждают разные подходы к лечению острого холецистита у соматически отягощенных пациентов [6, 7, 13]. В описанный период в клинике проводили лечение 16 пациентам с острым калькулезным холециститом. У девяти из них провели консервативное лечение с эффектом, в шести случаях была выполнена лапароскопическая холецистэктомия. У шести пациентов имело место сочетание острого холецистита с вирусной пневмонией со степенью поражения легочной паренхимы КТ-3-4. Этим пациентам было выполнено дренирование желчного пузыря под УЗ-контролем. В дальнейшем гладкое течение послеоперационного периода, холецистостома была удалена в срок от 9 до 18 суток после операции.

По поводу острого деструктивного панкреатита были пролечены 12 пациентов. Диагноз был подтвержден с помощью МСКТ, во всех случаях было выполнено чрескожное пункционное дренирование жидкостных скоплений под УЗ-контролем. В одном случае имел место летальный исход у пациента с деструктивным панкреатитом и тяжелой (КТ-4) вирусной пневмонией.

У девяти пациентов были проведены вмешательства по поводу острой кишечной непроходимости на фоне вирусной пневмонии со степенью поражения легочной паренхимы КТ-3-4. В пяти случаях непроходимость имела спаечную природу, было выполнено рассечение спаек (в двух случаях лапароскопически). В четырех случаях непроходимость была вызвана опухолью прямой и сигмовидной кишки. Этим пациентам в двух случаях, ввиду наличия признаков перфорации опухоли, была выполнена резекция сигмовидной кишки с выведением стомы, а в двух остальных случаях - выведение «двухствольных» колостом. В раннем послеоперационном периоде умерли два пациента с тяжелой вирусной пневмонией (КТ-3-4) и сопутствующей патологией.

По поводу ущемленных грыж было прооперировано три пациента. В одном случае имела место ущемленная паховая грыжа (выполнено грыжесечение с последующим выздоровлением), в другом - ущемленная гигантская вентральная грыжа у пациентки с многочисленными сопутствующими заболеваниями (выполнено грыжесечение с летальным исходом ввиду тяжелого коморбидного фона), в третьем случае - ущемленная диафрагмальная грыжа у пациентки на третьи сутки после кесарева сечения. Пациентке были выполнены лапаротомия, низведение желудка в брюшную полость, ушивание дефекта диафрагмы. Послеоперационный период протекал гладко, пациентка была выписана.

За период перепрофилирования трех пациентов прооперировали по поводу перфоративных язв желудка и двенадцатиперстной кишки. Во всех наблюдениях ушивание язв выполняли «открытым» способом. В одном случае у 92-летней пациентки имел место распространенный перитонит, в послеоперационном периоде возникла несостоятельность ушитой язвы. Были выполнены две релапаротомии, пациентка скончалась на восьмые сутки с момента поступления в стационар.

У 34 больных имели место язвы желудка и двенадцатиперстной кишки, осложненные кровотечением. Экстренная фиброгастродуоденоскопия (ФГДС) у пациентов c COVID-19 несет в себе риск инфицирования эндоскопической бригады, поэтому некоторые специалисты рекомендуют выполнять ее только по особым показаниям в течение 24 ч с момента поступления больного в стационар $[7,14]$. В нашем случае всем пациентам была выполнена экстренная ФГДС в течение первых трех ч с момента

Таблица 2. Распределение пациентов по степени тяжести пневмонии

\begin{tabular}{|l|c|c|}
\hline \multicolumn{1}{|c|}{ Степень тяжести пневмонии } & Число пациентов & \% от общего числа пациентов \\
\hline COVID-19 без пневмонии & 10 & 2 \\
\hline Пневмония КT-1 & 118 & 24,5 \\
\hline Пневмония KT-2 & 228 & 47,4 \\
\hline Пневмония КT-3 & 85 & 17,6 \\
\hline Пневмония КT-4 & 41 & 8,5 \\
\hline Всего: & 482 & 100 \\
\hline
\end{tabular}


поступления. Эндоскопическое исследование было закончено первичным гемостазом, однако рецидив кровотечения возник у 12 пациентов. Повторный эндоскопический гемостаз был эффективен у 11 из них. Один пациент после повторного рецидива был экстренно прооперирован: выполнены лапаротомия, дуоденотомия, прошивание кровоточащей язвы с последующим полным выздоровлением.

Некоторой особенностью описанного в статье периода можно считать практически одновременное поступление двух пациентов со спонтанным разрывом селезенки. Из симптомов заболевания пациенты жаловались на боль в левой половине грудной клетки, возникающую при кашле. В обоих случаях диагноз был установлен при УЗ-исследовании. Объем подкапсульной гематомы у пациентов составил 55 и 120 мл. Нами была выбрана консервативно-выжидательная тактика. В первые сутки отрицательной динамики отмечено не было, а на вторые сутки было выявлено увеличение гематомы до 150 и 220 мл соответственно с появлением полоски свободной

Таблица 3. Операции, выполненные в ГКБ № 15 имени О. М. Филатова в условиях перепрофилирования в инфекционный стационар для пациентов с COVID-19

\begin{tabular}{|c|c|c|c|}
\hline Название операции & Апрель 2020 г. & Май 2020 г. & $\begin{array}{c}\text { Прирост числа } \\
\text { операций, } \\
\%\end{array}$ \\
\hline \multicolumn{4}{|l|}{ Общехирургический профиль } \\
\hline Аппендэктомия & 9 & 19 & \\
\hline Ампутация (пальцев, голени, бедра) & 14 & 25 & \\
\hline Резекция сигмовидной кишки на фоне перитонита & 3 & 1 & \\
\hline Лапаротомия, тромбэктомия из верхнебрыжеечной артерии при мезентериальном тромбозе & 2 & 2 & \\
\hline Рассечение спаек при острой кишечной непроходимости & 3 & 2 & \\
\hline Спленэктомия & 2 & 1 & \\
\hline Лапароскопическая холецистэктомия (ЛХЭ) & 0 & 6 & \\
\hline Микрохолецистостомия & 2 & 4 & \\
\hline Операции при ущемленных грыжах & 0 & 2 & \\
\hline Ушивание перфоративных язв & 0 & 2 & \\
\hline Чрескожное чреспеченочное дренирование желчных протоков & 1 & 0 & \\
\hline Эндоскопическая ретроградная папиллотомия & 5 & 5 & \\
\hline Вскрытие флегмоны & 14 & 24 & \\
\hline ВСЕГО: & 55 & 93 & 69,09 \\
\hline \multicolumn{4}{|l|}{ Кардиохирургия и сосудистый профиль } \\
\hline Ангиопластика коронарных артерий & 13 & 21 & \\
\hline Тромбэктомия из артерий нижних конечностей & 12 & 21 & \\
\hline Установка кава-фильтра & 1 & 3 & \\
\hline Формирование артерио-венозной фистулы & 1 & 5 & \\
\hline Протезирование аорты & 1 & 0 & \\
\hline Имплантация кардиостимулятора & 0 & 4 & \\
\hline Аортокоронарное шунтирование (АКШ) & 0 & 1 & \\
\hline Протезирование клапанов сердца & 0 & 2 & \\
\hline Стентирование артерий нижних конечностей & 0 & 2 & \\
\hline Имплантация сосудистого катетера & 6 & 4 & \\
\hline ВСЕГО: & 34 & 63 & 85,29 \\
\hline \multicolumn{4}{|l|}{ Травматологический профиль } \\
\hline Остеосинтез & 8 & 15 & \\
\hline Транспедикулярная фиксация позвонков & 0 & 1 & \\
\hline Эндопротезирование тазобедренного сустава при переломе & 0 & 3 & \\
\hline ВСЕГО: & 8 & 19 & 137,5 \\
\hline \multicolumn{4}{|l|}{ Нейрохирургический профиль } \\
\hline Трепанация черепа при внутричерепных гематомах & 0 & 6 & \\
\hline ВСЕГО: & 0 & 6 & \\
\hline \multicolumn{4}{|l|}{ Урологический профиль } \\
\hline Нефростомия & 5 & 10 & \\
\hline ВСЕГО: & 5 & 10 & 100 \\
\hline $\begin{array}{l}\text { Прочие (диагностическая лапароскопия, первичная хирургическая обработка ран, } \\
\text { трахеостомия, дренирование плевральной полости и т. п.) }\end{array}$ & 92 & 98 & \\
\hline итого: & 194 & 289 & 48,96 \\
\hline
\end{tabular}


жидкости в брюшной полости. Обоих пациентов прооперировали в объеме спленэктомии с последующим полным выздоровлением.

У двух больных, поступивших в клинику с тяжелой пневмонией (КТ-3-4), проводили ИВЛ ввиду дыхательной недостаточности, у них был обнаружен пневмоперитонеум на фоне пневмомедиастинума. Дежурный хирург заподозрил перфорацию полого органа, однако во время операции повреждений органов брюшной полости обнаружено не было. Оба пациента скончались при нарастающих явлениях острой дыхательной и полиорганной недостаточности. Описанные случаи требуют дальнейшего подробного анализа и оценки.

Следует особо обсудить пациентов с мезентериальным тромбозом. Летальность в этой группе превышает 75\% даже в «мирное» время [15]. В описанной нами группе таких пациентов было восемь. В четырех случаях больные поступили с клинической картиной перитонита, и во время экстренной операции был обнаружен тотальный некроз тонкой кишки и правой половины толстой кишки у четырех больных были выполнены тромбэктомия из верхнебрыжеечной артерии с резекцией тонкой кишки. В одном случае тромбэктомия была выполнена эндоваскулярно. Однако, несмотря на назначения антикоагулянтов в лечебных дозах, у всех пациентов в раннем послеоперационном периоде наступили ретромбоз, некроз оставшейся части тонкой кишки и летальный исход.

Представленные данные свидетельствуют о значительном увеличении числа экстренных и срочных оперативных вмешательств в мае 2020 г. по сравнению с апрелем 2020 г. В апреле 2020 г. в клинику в основном поступали пациенты с вирусной пневмонией, вызванной COVID-19, а экстренные хирургические заболевания были обнаружены лишь у некоторых из них. В мае 2020 г. ситуация изменилась. В связи с тем, что в Москве для лечения пациентов с COVID-19 открыли несколько других крупных клиник, в ГКБ № 15 им. О. М. Филатова стали поступать пациенты, изначально имевшие острую хирургическую патологию на фоне COVID-19.

Накопленный клинический опыт позволяет констатировать, что принципиальных изменений в тактике ведения больных с острой хирургической патологией нет. Однако в условиях наличия у пациента, помимо острого хирургического заболевания, COVID-19 и вирусной пневмонии различной степени тяжести необходимо учитывать повышенный риск как бактериальных, так и тромбоэмболических осложнений.

На основании неполных двух месяцев работы сложно делать выводы об особенностях течения острой хирургической патологии на фоне вирусной пневмонии. Однако уже очевидно, что на пике заболеваемости COVID-19 при правильной организации хирургической помощи в инфекционном стационаре для пациентов с новой коронавирусной инфекцией можно соблюсти баланс между уменьшением объема хирургической помощи до уровня неотложной с одной стороны и риском заражения медицинского персонала с другой.

\section{ВЫВОДЫ}

В условиях наличия у пациента, помимо острого хирургического заболевания, COVID-19 и вирусной пневмонии различной степени тяжести необходимо учитывать повышенный риск как бактериальных, так и тромбоэмболических осложнений. Среди особенностей хирургической помощи в инфекционном стационаре для пациентов с COVID-19 можно назвать необходимость строгого соблюдения эпидемиологического режима, минимизации численности персонала в операционной, возможной минимизации числа оперативных пособий и сокращения их длительности. Наиболее важной задачей в этих условиях является безопасность персонала.

\section{Литература}

1. Приказ Министерства здравоохранения РФ от 19 марта 2020 г. № 198н «О временном порядке организации работы медицинских организаций в целях реализации мер по профилактике и снижению рисков распространения новой коронавирусной инфекции COVID-19» (2020).

2. Ti LK, Ang LS, Foong TW, Ng BSW. What we do when a COVID-19 patient needs an operation: operating room preparation and guidance. Can J Anaesth. 2020; 67 (6): 756-8. DOI: 10.1007/ s12630-020-01617-4.

3. Lenzen-Schulte M. COVID-19: Chirurgie in Zeiten der Pandemie. Dtsch Arztebl. 2020; 117 (18): A-940/B-793.

4. Akladios $\mathrm{C}$, Azais $\mathrm{H}$, Ballester $\mathrm{M}$, et al. Recommendations for the surgical management of gynecological cancers during the COVID-19 pandemic - FRANCOGYN group for the CNGOF. J Gynecol Obstet Hum Reprod. 2020; 49 (6): 101729. DOI:10.1016/j.jogoh.2020.101729.

5. Smith D, Montagne J, Raices M, et al. Tracheostomy in the intensive care unit: Guidelines during COVID-19 worldwide pandemic [published online ahead of print, 2020 Jun 1]. Am J Otolaryngol. 2020; 41 (5): 102578. doi:10.1016/j.amjoto.2020.102578.

6. Pryor A. SAGES and EAES recommendations regarding surgical response to COVID-19 crisis. Society of American Gastrointestinal and Endoscopic Surgeons, 2020 March 29. Available at: https:// www.sages.org/recommendations-surgical-response-covid-19/.

7. Sultan S, Lim JK, Altayar O, et al. AGA Institute Rapid Recommendations for Gastrointestinal Procedures During the

COVID-19 Pandemic [published online ahead of print, 2020 Mar 31]. Gastroenterology. 2020; S0016-5085 (20) 30458-3. DOI: 10.1053/j.gastro.2020.03.072.

8. COVID 19: Elective Case Triage Guidelines for Surgical Care, Emergency General Surgery. American College of Surgeons. 2020 March 24. Available at: https://www.facs.org/covid-19/ clinical-guidance/elective-case.

9. Updated General Surgery Guidance on COVID-19. 2020 April 6. Available at: https://www.augis.org/wp-content/ uploads/2020/04/2nd-Update-Intercollegiate-General-SurgeryGuidance-on-COVID-19-6-April-.._.pdf.

10. Готье С. В, Ревишвили А. Ш., Пушкарь Д. Ю. и др. Методические рекомендации «Экстренная хирургическая помощь в условиях COVID-19». М., 2020.

11. Временные методические рекомендации: профилактика, диагностика и лечение новой коронавирусной инфекции (COVID 19), версия 6. Москва. 28.04.2020.

12. Schreckenbach T, Fritsch N, Lahrsow M. SARS-CoV-2 pandemic a complicated case of appendicitis. Dtsch Arztebl Int. 2020; 117: 364. DOI: 10.3238/arztebl.2020.0364.

13. Ambe PC, Kaptanis S, Papadakis M, Weber SA, Jansen S, Zirngibl $\mathrm{H}$. The Treatment of Critically III Patients With Acute Cholecystitis. Dtsch Arztebl Int. 2016; 113 (33-34): 545-51. DOI: 10.3238/arztebl.2016.0545.

14. Lau JYW, Yu Y, Tang RSY, et al. Timing of Endoscopy for Acute Upper Gastrointestinal Bleeding. N Engl J Med. 2020; 382 (14): 
1299-308. DOI: 10.1056/NEJMoa1912484.

15. Bala M, Kashuk J, Moore EE, et al. Acute mesenteric ischemia: guidelines of the World Society of Emergency Surgery. World J Emerg Surg. 2017; 12:38. DOI: 10.1186/s13017-017-0150-5.

\section{References}

1. Prikaz Ministerstva zdravoohranenija RF ot 19 marta $2020 \mathrm{~g}$. № 198n "O vremennom porjadke organizacii raboty medicinskih organizacij v celjah realizacii mer po profilaktike i snizheniju riskov rasprostranenija novoj koronavirusnoj infekcii COVID-19" (2020). Russian.

2. Ti LK, Ang LS, Foong TW, Ng BSW. What we do when a COVID-19 patient needs an operation: operating room preparation and guidance. Can J Anaesth. 2020; 67 (6): 756-8. DOI: 10.1007/ s12630-020-01617-4.

3. Lenzen-Schulte M. COVID-19: Chirurgie in Zeiten der Pandemie. Dtsch Arztebl. 2020; 117 (18): A-940/B-793.

4. Akladios $\mathrm{C}$, Azais $\mathrm{H}$, Ballester $\mathrm{M}$, et al. Recommendations for the surgical management of gynecological cancers during the COVID-19 pandemic - FRANCOGYN group for the CNGOF. J Gynecol Obstet Hum Reprod. 2020; 49 (6): 101729. DOI:10.1016/j.jogoh.2020.101729.

5. Smith D, Montagne J, Raices M, et al. Tracheostomy in the intensive care unit: Guidelines during COVID-19 worldwide pandemic [published online ahead of print, 2020 Jun 1]. Am J Otolaryngol. 2020; 41 (5): 102578. doi:10.1016/j.amjoto.2020.102578.

6. Pryor A. SAGES and EAES recommendations regarding surgical response to COVID-19 crisis. Society of American Gastrointestinal and Endoscopic Surgeons, 2020 March 29. Available at: https:// www.sages.org/recommendations-surgical-response-covid-19/.

7. Sultan S, Lim JK, Altayar O, et al. AGA Institute Rapid Recommendations for Gastrointestinal Procedures During the COVID-19 Pandemic [published online ahead of print, 2020 Mar 31]. Gastroenterology. 2020; S0016-5085 (20) 30458-3. DOI: 10.1053/j.gastro.2020.03.072

8. COVID 19: Elective Case Triage Guidelines for Surgical Care, Emergency General Surgery. American College of Surgeons. 2020 March 24. Available at: https://www.facs.org/covid-19/ clinical-guidance/elective-case.

9. Updated General Surgery Guidance on COVID-19. 2020 April 6. Available at: https://www.augis.org/wp-content/ uploads/2020/04/2nd-Update-Intercollegiate-General-SurgeryGuidance-on-COVID-19-6-April-.._.pdf.

10. Gote SV, Revishvili ASh, Pushkar DJu i dr. Metodicheskie rekomendacii "Jekstrennaja hirurgicheskaja pomoshh' v uslovijah COVID-19". M., 2020. Russian.

11. Vremennye metodicheskie rekomendacii: profilaktika, diagnostika i lechenie novoj koronavirusnoj infekcii (COVID 19), versija 6. Moskva. 28.04.2020. Russian.

12. Schreckenbach T, Fritsch N, Lahrsow M. SARS-CoV-2 pandemic a complicated case of appendicitis. Dtsch Arztebl Int. 2020; 117: 364. DOI: 10.3238/arztebl.2020.0364.

13. Ambe PC, Kaptanis S, Papadakis M, Weber SA, Jansen S, Zirngibl H. The Treatment of Critically III Patients With Acute Cholecystitis. Dtsch Arztebl Int. 2016; 113 (33-34): 545-51. DOI: 10.3238/arztebl.2016.0545.

14. Lau JYW, Yu Y, Tang RSY, et al. Timing of Endoscopy for Acute Upper Gastrointestinal Bleeding. N Engl J Med. 2020; 382 (14): 1299-308. DOI: 10.1056/NEJMoa1912484.

15. Bala M, Kashuk J, Moore EE, et al. Acute mesenteric ischemia: guidelines of the World Society of Emergency Surgery. World $J$ Emerg Surg. 2017; 12:38. DOI: 10.1186/s13017-017-0150-5. 